\title{
In-hospital end-of-life decisions after new traumatic spinal cord injury in the Netherlands
}

\author{
R Osterthun ${ }^{1,2}$, FWA van Asbeck ${ }^{3,4}$, JHB Nijendijk ${ }^{5}$ and MWM Post ${ }^{2,4}$
}

Study design: Explorative retrospective files study.

Objectives: To document end-of-life decisions (ELDs) in in-hospital deaths after new traumatic spinal cord injury (TSCI).

Setting: The Netherlands.

Methods: Discharge letters concerning patients with TSCI discharged from Dutch acute hospitals in 2010 were analysed. Data were extracted on survival, personal and lesion characteristics, comorbidities, other injuries, preexisting spinal stenosis, stabilising surgery, length of hospital stay and the presence and types of ELDs. Characteristics of deceased patients and survivors were compared using $\chi^{2}$ and $T$-tests. Characteristics of the deceased patients and ELDs were further explored.

Results: A total of 185 patients with new TSCI were identified. Twenty-six patients were excluded as their survival status at discharge was unknown-for example, because of discharge to another hospital without information about their final discharge. Thirty of the remaining 159 patients died during their initial hospital stay (18.9\%). Deceased patients were older and had more often high cervical and motor complete injuries than survivors. The circumstances of death were sparsely documented, and in nine cases, it was not possible to determine the absence or the presence of an ELD. ELDs were reported in 19 deaths (63.3\%). All were non-treatment decisions, and almost all (89.5\%) were decisions of withdrawal of treatment. There were no cases of documented euthanasia or physician-assisted suicide.

Conclusion: ELDs were reported in the majority of in-hospital deaths after new $\mathrm{TSCl}$ in the Netherlands (63.3\%), and all were nontreatment decisions.

Spinal Cord (2016) 54, 1025-1030; doi:10.1038/sc.2016.37; published online 12 April 2016

\section{INTRODUCTION}

Survival after traumatic spinal cord injury (TSCI) has improved considerably after World War II. In the past decades, the largest improvements in survival have been achieved in the first year after the injury. ${ }^{1}$ Several initiatives to improve the acute care seem to have contributed to this trend. ${ }^{2}$ As more people with a TSCI nowadays survive the accident and emergency care, this includes cases with a poor long-term prognosis. In these cases, it is likely that patients, family members and health-care professionals will make considerations whether starting or continuing treatment would make sense considering the future quality of life of the patient. These considerations may lead to decisions concerning the end of life and thereby obviously go hand in hand with ethical dilemmas.

End-of-life decisions (ELDs) can be defined as all decisions made by a physician, either with the intention of shortening the patient's life or knowing that this decision may have a potentially life-shortening effect. $^{3}$ There is no standard and widely agreed terminology for ELDs. ${ }^{3}$ However, categories as displayed in Table 1 are frequently used to describe ELDs. The most radical form of an ELD is the decision for euthanasia. The definition of euthanasia is still debated. The Dutch definition of euthanasia is similar to the definition of the European Association for Palliative Care and is adopted in our paper (Table 1). ${ }^{4}$
In the Netherlands, general demands of carefulness with regard to patient-physician relationships are regulated by law, the Act on the Medical Treatment Agreement (WGBO). For example, the WGBO states that the patient should be well informed about his situation, there should be dialogue with a representative in case of incompetence of the patient and that procedures, decisions and considerations should be well documented in the patients' file. Furthermore, as a principle, the consent of the patient is required for (non-)treatment decisions. Exceptions are, however, possible-for example, in case of medical futility.

The active ending of life is not considered as regular medical practice and is thus not incorporated in the WGBO. A separate law exists on this subject, the Dutch Termination of Life on Request and Assisted Suicide (Review Procedures) Act, which is also referred to as the Euthanasia law. ${ }^{5}$ In case of euthanasia and physician-assisted suicide, the attending physician has to act in accordance with six criteria of due care to qualify for a legal exception for homicide. These criteria concern the patient's request (it must be voluntary and well thought-out), the patient's suffering (it is unbearable and hopeless), full information provided to the patient, the absence of reasonable alternatives, the consultation of another physician and the applied method of ending life. To demonstrate their compliance, physicians

\footnotetext{
${ }^{1}$ Jeroen Bosch Hospital, Tolbrug Rehabilitation Centre, Groningen, The Netherlands; ${ }^{2}$ University of Groningen, University Medical Center Groningen, Center for Rehabilitation, Groningen, The Netherlands; ${ }^{3}$ De Hoogstraat Rehabilitation, Utrecht, The Netherlands; ${ }^{4}$ Brain Center Rudolf Magnus and Center of Excellence in Rehabilitation Medicine, University Medical Center Utrecht and De Hoogstraat Rehabilitation, Utrecht, The Netherlands and ${ }^{5}$ Rijndam Rehabilitation, Rotterdam, The Netherlands Correspondence: R Osterthun, Jeroen Bosch Hospital, Tolbrug Rehabilitation Centre, Henri Dunantstraat 7, 's-Hertogenbosch, NL5223GZ, The Netherlands. E-mail: R.Osterthun@Tolbrug.nl Received 28 September 2015; revised 11 February 2016; accepted 18 February 2016; published online 12 April 2016
} 


\section{Table 1 Categories of ELDs}

Euthanasia: a doctor intentionally killing a person by the administration of drugs, at that person's voluntary and competent request.

Physician-assisted suicide: a doctor intentionally helping a person to commit suicide by providing drugs for self-administration, with that person's voluntary and competent request.

Non-treatment decisions: decisions to withhold or withdraw potentially life-sustaining treatment.

Intensified pain and/or other symptom management by the administration of drugs in potentially life-shortening doses.

Abbreviation: ELD, end-of-life decision.

have to report euthanasia to a review committee. ${ }^{6}$ Doctors are prosecuted and can be punished if the legal exceptions are not met.

ELD making is extremely complex and variable on the part of health-care professionals, patients and families. Attitudes towards ELDs are determined by several factors, which on a societal level include culture, law and resources and on an individual level the prognosis, expected functional outcomes and opinions of patients and/ or family members. Some studies on ELDs in trauma patients, which also included patients with TSCI, found that a significant number of in-hospital deaths (42-61\%) were preceded by non-treatment decisions. ${ }^{7-10}$ Advanced age and comorbidities have been found to be associated with these decisions. ${ }^{7}$ A survey by Ball et al. ${ }^{11}$ on the clinicians' viewpoints towards the application end-of-life care for injured patients on intensive care units found that ELD making varied across several continents with regard to age and functional level for patients with a TSCI. However, irrespective of region, most respondents agreed that the level of SCI affected ELDs, with the absence of diaphragm function as being the most important. ${ }^{11}$

Several studies have been performed on in-hospital death after new TSCI. ${ }^{12-19}$ However, the only studies on ELDs after new TSCI we could find were studies on ethical considerations, including case reports, ${ }^{20-22}$ and the aforementioned study on clinicians' viewpoints towards non-treatment decisions. ${ }^{11}$ Information on the occurrence of ELDs and characteristics of the patients involved is thus lacking. It is further unclear whether results from general trauma studies apply to patients with a new TSCI. Within the group of trauma patients, patients with a TSCI have specific characteristics that justify a separate appraisal. Information on ELDs after new TSCI will also help understanding mortality figures ${ }^{10,12}$ and will give insight in rationales of these decisions.

As little is known on in-hospital ELDS after new TSCI, we performed an explorative study to document ELDs in in-hospital deaths after TSCI in the Netherlands. The main study objectives were to describe the characteristics of deceased patients, the extent to which ELDs occur and the different types of ELDs.

\section{METHODS}

This study was a secondary analysis of a hospital-based study on the incidence of TSCI in the Netherlands. ${ }^{23}$

\section{Study population}

The study population consisted of patients with a new TSCI who were admitted to Dutch acute hospitals and discharged with a known survival status in 2010. TSCI was defined as a newly acquired traumatic transverse lesion of the spinal cord or cauda equina, resulting in loss of motor, sensory, bladder or bowel function below the level of the lesion and lasting $>2$ weeks.

\section{Procedure}

Records of patients discharged from hospitals in 2010 with the International Classification of Diseases Ninth edition (ICD-9) code 806 (fracture of the spine with injury of the spinal cord) or 952 (injury to the spinal cord without apparent spinal fracture) were retrieved from the Landelijke Medische
Registratie (National Medical Registration), the national database of patients hospitalised in an acute care hospital (trauma centre or general hospital) in the Netherlands. After removal of duplicate records, involved hospitals were requested to send us the anonymised discharge letters concerning these patients. ${ }^{23}$

First, these letters were analysed for the presence of TSCI. If so, survival status at discharge and, if applicable, information on the ELD or cause of death were extracted from the discharge letter. Patients were excluded if their survival status at discharge was unknown-for example, because of discharge to another hospital without information about the final discharge. To get an impression of the involvement of the patient in the ELD, the discharge letters were searched for the presence of advance directives, statements on the level of consciousness (conscious, confused or unconscious) of the patient and agreement on the ELD between physician and patient and/or family.

Finally, data were extracted on sex, age, aetiology of injury, other injuries, comorbidities, lesion characteristics, preexisting spinal stenosis, stabilising surgery and length of hospital stay (LOHS). The LOHS was set at 0 if the patient had died or was transferred to another hospital on the day of admission.

Approval by a Medical Ethics Review Committee was not required because discharge letters were anonymised and no patients were at risk in the study.

\section{Instruments}

ELDs were categorised as noted in Table 1. If terms such as 'palliative management' or 'palliative care' were used, this was also recorded. However, as palliative care intends neither to hasten nor to postpone death, ${ }^{24}$ this was not considered as an ELD.

Having other injuries was defined as the presence of injuries other than the TSCI on admission. They were scored according to the six-point Abbreviated Injury Scale ${ }^{25}$ and considered to be relevant when at least one grade 2 (moderate injury) was present. Comorbidities were defined as the presence of one or more other diseases according to Charlson Index. ${ }^{26}$

Lesion characteristics were assessed according to the International Standards for Neurological Classification of SCI. ${ }^{27}$ The American Spinal Injury Association Impairment Scale classifications A and B were considered motor complete and the classifications $\mathrm{C}$ and D were considered motor incomplete. Neurological lesion level was defined as the lowest intact motor and sensory level. Levels of injury C1-C8 were defined as tetraplegia, C1-C4 as high tetraplegia, C5-C8 as low tetraplegia and below $\mathrm{C} 8$ as paraplegia.

\section{Analyses}

Analyses were performed using IBM SPSS Statistics V.19 (IBM, Armonk, NY, USA). Characteristics of deceased patients and survivors were compared using $\chi^{2}$ test and independent $T$-test. The retrieved information on ELDs was further categorised.

\section{RESULTS}

A total of 185 patients with TSCI were identified. Survival status was known in 159 patients. The other 26 patients were excluded from the analyses. The main reason for an unknown survival status was discharge to another hospital ward or acute care hospital. In the excluded group, more patients underwent a stabilising operation (84.6\% versus $53.5 \% ; P=0.003$ ) than in the included group. Other characteristics did not significantly differ between both groups. 
Table 2 Characteristics of deceased patients and survivors

\begin{tabular}{|c|c|c|c|c|c|}
\hline Characteristics & $\begin{array}{c}\text { Survivors, \% } \\
(\mathrm{N}=129)\end{array}$ & $\begin{array}{c}\text { Deceased, \% } \\
\quad(\mathrm{N}=30)\end{array}$ & $\begin{array}{c}\text { Total, } \% \\
(\mathrm{~N}=159)\end{array}$ & $\chi^{2}$ & P-value \\
\hline \multicolumn{6}{|l|}{ Sex } \\
\hline Male & 73.6 & 76.7 & 74.2 & 0.1 & 0.733 \\
\hline Female & 26.4 & 23.3 & 25.8 & & \\
\hline \multicolumn{6}{|l|}{ Age at injury } \\
\hline$<65$ years & 65.1 & 13.3 & 55.3 & 26.4 & 0.000 \\
\hline $65+$ years & 34.9 & 86.7 & 44.7 & & \\
\hline \multicolumn{6}{|l|}{ Cause of injury } \\
\hline Sports & 14.0 & 13.3 & 13.8 & 0.6 & 0.898 \\
\hline Traffic & 22.5 & 16.7 & 21.4 & & \\
\hline Fall & 52.7 & 56.7 & 53.5 & & \\
\hline Other & 10.9 & 13.3 & 11.3 & & \\
\hline \multicolumn{6}{|l|}{ Level of injury } \\
\hline $\mathrm{C} 1-\mathrm{C} 4$ & 30.3 & 63.3 & 35.7 & 12.3 & 0.002 \\
\hline $\mathrm{C} 5-\mathrm{C} 8$ & 25.2 & 10.0 & 22.7 & & \\
\hline T1-S5 & 36.1 & 16.7 & 33.0 & & \\
\hline Tetraplegia unspecified ${ }^{a}$ & 8.4 & 10.0 & 8.6 & & \\
\hline \multicolumn{6}{|l|}{ Completeness of injury $(7)^{b}$} \\
\hline Motor complete & 32.3 & 56.0 & 36.2 & 5.1 & 0.024 \\
\hline Motor incomplete & 67.7 & 44.0 & 63.8 & & \\
\hline \multicolumn{6}{|l|}{ Comorbidities $(30)^{b}$} \\
\hline Yes & 47.1 & 72.0 & 51.9 & 5.0 & 0.025 \\
\hline No & 52.9 & 28.0 & 48.1 & & \\
\hline \multicolumn{6}{|l|}{ Other injuries $(5)^{\mathrm{b}}$} \\
\hline Yes & 28.2 & 16.7 & 26.0 & 1.7 & 0.195 \\
\hline No & 71.8 & 83.3 & 74.0 & & \\
\hline \multicolumn{6}{|c|}{ Preexisting spondylarthropathy $(2)^{\mathrm{b}}$} \\
\hline Yes & 41.4 & 48.3 & 42.7 & 0.5 & 0.499 \\
\hline No & 58.6 & 51.7 & 57.3 & & \\
\hline \multicolumn{6}{|l|}{ Stabilising operation } \\
\hline Yes & 58.1 & 33.3 & 53.5 & 6.0 & 0.014 \\
\hline No & 41.9 & 66.7 & 46.5 & & \\
\hline
\end{tabular}

${ }^{a} N=15$; not included in $\chi^{2}$ analysis.

bMissing.

The mean LOHS was 25.3 days (s.d. 28.5; median 18.0; range $0-136)$. Mean age at injury was 57.0 years (s.d. 22.2; median 62.0; range 13-100).

A total of 30 of 159 patients (18.9\%) died during their hospital stay. Mean time between onset of injury and death was 14.1 days (s.d. 32.6; median 4, range 0-136). Mean age of deceased patients was 73.6 years (s.d. 19.4; median 79.5; range 13-100) versus 52.3 years (s.d. 21.1; median 56; range 14-91) of survivors. Other characteristics of deceased patients and survivors are displayed in Table 2.

In Figure 1, the age distribution is displayed for deceased patients and survivors. Characteristics of deceased patients are displayed in more detail in Table 3. The information in Table 3 has been sorted primarily by the information on the ELDs and secondarily by age. As can be seen in this table, $50 \%$ of deceased patients were aged $80+$ years, and $86.7 \%$ of them were aged $65+$ years.

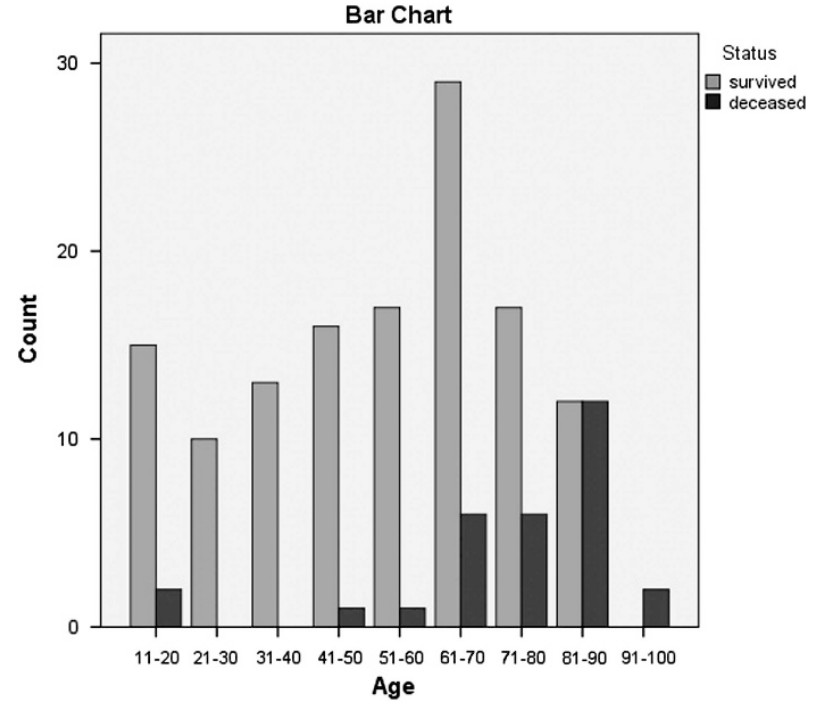

Figure 1 Age distribution for deceased patients and survivors.

\section{End-of-life decisions}

ELDs were reported in 19/30 (63.3\%) of deceased patients (Table 3). In the other 11 cases, the letter was incomplete (3), there was no documented ELD (6) or the circumstances of death indicated that there had been no ELD (2). All ELDs consisted of non-treatment decisions. There were no cases of euthanasia or physician-assisted suicide. Almost all non-treatment decisions consisted of a withdrawal of treatment $(89.4 \%)$. In half of the cases with no documented ELD, the letter noted the start of palliative care. We did not find clues for the application of intensified pain and/or other symptom management by the administration of drugs in potentially life-shortening doses.

Mean age of patients with ELDs was 72.6 years (s.d. 19.4; median 77.0; range 13-100). Injuries were high cervical (C1-C4) in about three-quarters and motor complete in about half of the cases. Comorbidities were present in $63.2 \%$ (10.5\% unknown). Other injuries were present in $10.5 \%$, and all consisted of at least brain injury. Patients with ELDs died on average 18.6 days (s.d. 40.3; median 4; range $0-136)$ after the onset of the SCI. A total of $68.4 \%$ patients with ELDs were unconscious or confused during the decision. In $73.7 \%$ of cases, it had been mentioned in the discharge letter that the decision was made together with the patient or its family. In the other seven cases, it was not mentioned. There were no documented advance directives. In nine cases, it had been mentioned that the ELD conformed to the wish of the patient, either formulated by the patient or by the family. Some quotes from the discharge letters on ELDs are displayed in Table 4.

\section{DISCUSSION}

This explorative study was a secondary analysis of a hospital-based study on the incidence of TSCI in the Netherlands in 2010 and is, as far as we know, the first study to focus on in-hospital ELDs after new TSCI. We found that 30 of the 159 patients died during their initial hospital stay. Patients who deceased were considerably older and had more comorbidities than patients who survived. Furthermore, their injuries were more frequently high cervical and motor complete.

We found that ELDs were reported in the majority of deaths (19/30, 63.3\%). All ELDs consisted of non-treatment decisions. There were no cases of euthanasia or physician-assisted suicide. 
Table 3 Deceased patients $(N=30)$

\begin{tabular}{|c|c|c|c|c|c|c|c|c|c|c|}
\hline Case & Age & Sex & Level of injury & Completeness & Comorbidity & Other injury & LOHS & Documented ELD (relevant circumstances) & Conscious & Agreement with \\
\hline 1 & 13 & M & $\mathrm{C} 1-\mathrm{C} 4$ & $A$ or $B$ & No & Yes & 3 & Withdrawal of treatment & No & Family \\
\hline 2 & 44 & M & T1-S5 & Unkown & No & Yes & 7 & Withdrawal of treatment & No & Familya \\
\hline 3 & 52 & M & $\mathrm{C} 1-\mathrm{C} 4$ & $A$ or $B$ & No & No & 2 & Withdrawal of treatment & No & Family \\
\hline 4 & 66 & M & $\mathrm{C} 1-\mathrm{C} 4$ & $A$ or $B$ & No & No & 128 & Withdrawal of treatment & Yes & Patient and family ${ }^{\mathrm{a}}$ \\
\hline 5 & 67 & M & $\mathrm{C} 1-\mathrm{C} 4$ & Unkown & Yes & No & 4 & Withdrawal of treatment & No & Not mentioned \\
\hline 6 & 68 & M & $\mathrm{C} 1-\mathrm{C} 4$ & Unkown & No & Yes & 12 & Withdrawal of treatment & No & Familya \\
\hline 7 & 70 & M & $\mathrm{C} 1-\mathrm{C} 4$ & $C$ or $D$ & Yes & No & 136 & Withdrawal of treatment (pneumonia) & Confused & Not mentioned \\
\hline 8 & 76 & M & $\mathrm{C} 1-\mathrm{C} 4$ & $A$ or $B$ & Yes & Unkown & 2 & Withdrawal of treatment & No & Not mentioned \\
\hline 9 & 77 & M & $\mathrm{C} 1-\mathrm{C} 4$ & $A$ or $B$ & Unknown & No & 1 & Withdrawal of treatment & No & Familya \\
\hline 10 & 77 & M & $\mathrm{C} 1-\mathrm{C} 4$ & $A$ or $B$ & Yes & No & 2 & Withdrawal of treatment ${ }^{\mathrm{b}}$ & No & Family $^{a}$ \\
\hline 11 & 79 & M & $\mathrm{C} 1-\mathrm{C} 4$ & $A$ or $B$ & Yes & No & 4 & Withdrawal of treatment ${ }^{\mathrm{b}}$ & No & Family \\
\hline 12 & 80 & M & $\mathrm{C} 1-\mathrm{C} 8$ & C or D & Yes & No & 8 & Withdrawal of treatment & Yes & Patient and familya \\
\hline 13 & 81 & M & $\mathrm{C} 1-\mathrm{C} 4$ & Unkown & Yes & No & 0 & Withdrawal of treatment & No & Not mentioned \\
\hline 14 & 84 & M & $\mathrm{C} 1-\mathrm{C} 4$ & $A$ or $B$ & Unkown & No & 1 & Withdrawal of treatment & Yes & Patient and family \\
\hline 15 & 85 & $\mathrm{~F}$ & T1-S5 & $A$ or $B$ & Yes & No & 18 & Withdrawal of treatment (pneumonia) & Yes & Patient and familya \\
\hline 16 & 85 & M & T1-S5 & $C$ or $D$ & Yes & No & 13 & Withdrawal of treatment & Yes & Patient and family ${ }^{\mathrm{a}}$ \\
\hline 17 & 87 & $\mathrm{~F}$ & $\mathrm{C} 1-\mathrm{C} 4$ & $C$ or $D$ & Yes & No & 2 & Withdrawal of treatment & Confused & Familya \\
\hline 18 & 89 & M & C5-C8 & $A$ or $B$ & Yes & No & 7 & Withholding of treatment (respiratory insufficiency) & Yes & Patient and family \\
\hline 19 & 100 & M & $\mathrm{C} 1-\mathrm{C} 4$ & C or D & Yes & No & 3 & Non-treatment, type unknown (pneumonia) & Confused & Not mentioned \\
\hline 20 & 83 & $\mathrm{~F}$ & $\mathrm{C} 1-\mathrm{C} 4$ & $A$ or $B$ & Yes & No & 1 & No documented ELD ${ }^{b}$ & & \\
\hline 21 & 84 & $\mathrm{~F}$ & C5-C8 & $C$ or $D$ & Yes & No & 9 & No documented ELD ${ }^{b}$ & & \\
\hline 22 & 95 & M & $\mathrm{C} 1-\mathrm{C} 8$ & $A$ or $B$ & Unkown & No & 5 & No documented ELD ${ }^{b}$ & & \\
\hline 23 & 68 & M & C5-C8 & $C$ or $D$ & Yes & No & 0 & No documented ELD & & \\
\hline 24 & 82 & $\mathrm{~F}$ & $\mathrm{C} 1-\mathrm{C} 4$ & $A$ or $B$ & No & No & 4 & No documented ELD (respiratory insufficiency) & & \\
\hline 25 & 86 & M & C1-C4 & C or $D$ & Yes & No & 2 & No documented ELD (respiratory insufficiency) & & \\
\hline 26 & 65 & $\mathrm{~F}$ & $\mathrm{C} 1-\mathrm{C} 8$ & C or D & Unkown & No & 27 & No ELD (death after resuscitation) & & \\
\hline 27 & 77 & $\mathrm{~F}$ & T1-S5 & C or D & Unkown & Yes & 13 & No ELD (brain herniation) & & \\
\hline 28 & 20 & M & T1-S5 & Unkown & No & Yes & 0 & Discharge letter incomplete & & \\
\hline 29 & 81 & M & $\mathrm{C} 1-\mathrm{C} 4$ & $C$ or $D$ & Yes & No & 3 & Discharge letter incomplete & & \\
\hline 30 & 86 & M & $\mathrm{C} 1-\mathrm{C} 4$ & $A$ or $B$ & Yes & No & 5 & Discharge letter incomplete & & \\
\hline
\end{tabular}

Abbreviations: ELD, end-of-life decision; F, female; LOHS, length of hospital stay; LOI, level of injury; M, male.

${ }^{a} E L D$ was the patient's wish, either formulated by the patient or by the family (no advance directives).

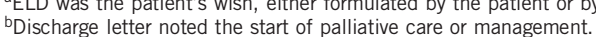

Comparing in-hospital ELDs between countries is difficult because of differences in, for example, culture, law and health-care systems. Furthermore, methodological differences between studies may determine mortality figures, as well as information on the application of ELDs. As this is the first study that has focussed primarily on this subject, and studies on in-hospital mortality after TSCI did not report on ELDs, ${ }^{12-19}$ it is not possible to compare our results with those of other studies in this diagnostic group. We found slightly more nontreatment decisions compared with studies on trauma patients $(42-61 \%) .^{7-10}$ In line with our results, Sise et al. ${ }^{7}$ concluded that non-treatment decisions were most commonly applied in frail elderly with comorbidities and less severe traumas. Because of the specific characteristics of patients with a TSCI, comparing with trauma patients is, however, simplistic.

Besides age and comorbidities, the level of the SCI seemed to be associated with ELDs after TSCI in our study. This appears to be in line with the European clinicians' viewpoints towards ELDs in the survey of Ball et al. ${ }^{11}$ That study showed that the level of injury and the absence of diaphragm function were considered to be important and that age became a more important factor above 65 years. They did not examine the viewpoint of clinicians on the influence of comorbidities or other injuries on ELDs. ${ }^{11}$
Because of ethical aspects and the complexity of ELDs, it is very difficult to make a value judgment on the application of ELDs. Several issues are relevant, such as the considerations, the capability of the patient to make an informed decision, the presence of an advance directive and the content of the information provided to the patient and family. Only limited information on these issues was available in the discharge letters, and therefore our study can only provide a general impression.

The LOHS of people with an ELD indicates that the timing of the ELD in our study was variable, ranging from 0 to 136 days. In general, there is a common belief that withholding of treatment is emotionally easier than withdrawing of treatment. This subtle difference may cause patients or families to feel pressure about a critical time window in which withholding of treatment is still possible. Most ELDs in our study were, however, decisions to withdraw treatment.

There is no consensus on the timing of a non-treatment decision on voluntary request. A minority of patients in our study were conscious, and some non-treatment decisions were performed on voluntary request. Being conscious may obviously not be equated with having the capacity of making an informed decision. The patient's capacity of decision making in the acute phase may be impaired by, for example, sedating medication, concomitant brain injury, mood disturbance or 
Table 4 Quotes from discharge letters

Case 1 (13 years, male, motor complete (high) tetraplegia, brain injury, no comorbidity): A complete spinal cord injury and severe postanoxic brain injury were diagnosed. Therefore, it was decided to stop the treatment. In the presence of his family, the respiratory support was stopped after which he died almost immediately.

Case 2 (44 years, male, unknown completeness, paraplegia, brain injury, no comorbidity): The patient became more and more respiratory and haemodynamically unstable. In consultation with the other physicians, a surgeon and a neurologist, treatment options were discussed with the family. The family knew that the patient's wish was not to be resuscitated if they ever had to make this choice. The patient had also indicated that he would not want to live with severe cognitive and physical impairments. After this conversation with the family, the treatment was withdrawn.

Case 3 (52 years, male, motor complete (high) tetraplegia, no other injury, no comorbidity): An EEG showed clues for postanoxic brain damage after resuscitation. Furthermore, the MRI of the cervical spinal cord showed severe damage of the myelum at $\mathrm{C} 2$. Because of the very poor prognosis, we decided to withdraw treatment in consultation with the family and the intensivist.

Case 10 (77 years, male, motor complete (high) tetraplegia, no other injury, hypertension): Considering the infaust prognosis and the patient's desire as expressed by the family that he would not want to live in such a situation, it was decided to stop the treatment that aimed at recovery and to start palliative sedation. The patient died four minutes after he was detubated.

Abbreviations: EEG, electroencephalogram; MRI, magnetic resonance imaging.

other emotional confounders that are related to the impact of an acute loss of future perspectives. ${ }^{20,22}$ Furthermore, information provided to the patient about the functional prognosis may be incomplete or coloured by the individual who provides the information. ${ }^{20,28}$ Considering these issues, it has been suggested that ELDs on voluntary request must be postponed to at least 2 years after the injury, as it may be impossible for the patient to realise what life will be like early after onset of the condition. ${ }^{20}$ On the other hand, postponing the ELD may conflict with the patients autonomy. ${ }^{29}$ The considerations on the need for postponing ELDs concern patients who want to end their life early after the injury without a profound overview of their perspectives. ${ }^{20,22}$ Most patients in our study who voluntarily requested to withdraw treatment were aged $80+$ years, but the sparsely documented circumstances of death in our study did not provide sufficient information for solid conclusions on this subject.

In cases of patients without the capacity of making an informed decision, the presence of an advance directive may facilitate decision making. In these cases, it would be interesting to get more insight into the considerations of the ELD. The adequate provision of information on perspectives to the patient and family will anyway remain important.

\section{Limitations}

The study population was relatively small. As discharge letters were retrieved by means of retrieved ICD-9 codes, cases with TSCI may have been missed, especially cases with severe other injuries. People who died at the scene were not included in the study. A small group of patients with an unknown survival status was excluded. The main characteristics of the excluded patients, however, did not differ from the included group.

Several limitations are the result of the retrospective nature of our study and the use of discharge letters. The usability of the letters was variable, as some letters contained limited information and some were incomplete. Owing to the sparsely documented circumstances of death, the number of ELDs may be underestimated, and details of individual cases have remained unknown.

For the purpose of this study, we have assumed that the ELDs have been correctly presented in the discharge letter. As the ELDs that are reported in this study are not controversial in the Netherlands, we do not expect under-reporting because of physician's reluctance to describe the critical events.

In six cases, the discharge letter noted the start of palliative care, two times after a pneumonia. In half of these cases, no further ELD was mentioned. In these cases, we are not sure about the correct usage of the term palliative care by the physician. Palliative care intends neither to hasten nor to postpone death according to the World Health Organisation definition. ${ }^{24}$ In a Belgian study, however, palliative sedation was found to be used as an umbrella term covering a large variety of practices that often coincided with co-intended or explicitly intended termination of life. ${ }^{3}$ This may also have been applicable to our results, although we did not find clues for the application of intensified pain and/or other symptom management by the administration of drugs in potentially life-shortening doses.

Because of the Dutch regulations, it seems unlikely that euthanasia has been performed without being reported. Performing euthanasia would also be illogical in cases in which withdrawing of treatment is possible, as the latter is more feasible and less aggravating. It may have, however, happened that ELDs, euthanasia included, were considered but postponed to the discharge destination.

\section{Implications}

This explorative study provides insight in the application of in-hospital ELDs after new TSCI in the Netherlands and showed that ELDs were reported in the majority of in-hospital deaths. As the ELDs were most prevalent in elderly patients, the number of elderly with a new TSCI is growing, ${ }^{23}$ and the acute care may further improve, the number of inhospital ELDs after TSCI can be expected to grow or at least remain substantial.

As this study showed that in-hospital ELDs after new TSCI were common, it is remarkable that little attention has been paid to this subject in the current literature. The use of a standard and widely agreed terminology for ELDs would be helpful for future studies. Including information on ELDs in future studies on in-hospital mortality will provide more insight into the application of ELDs across countries. Furthermore, providing this information may help in understanding mortality figures, ${ }^{10,12}$ although estimating to what extent mortality figures are influenced by ELDs will remain complex.

Larger, prospective studies focussing on ELDs in more detail can further contribute to the understanding of the application of ELDs. More specifically, information on the types of ELDs, characteristics of the involved patients, considerations, the timing, the capability of the patients to make an informed decision and the content of the information provided to the patient and family would be valuable. Gaining insight into the application of ELDs across countries may ultimately lead to a better substantiation of decisions. 
The frequent occurrence of ELDs supports the importance of standardisation of care around in-hospital ELDs after TSCI. This should, among other things, comprise a thorough provision of information on the prognosis and expected outcomes to the patient and family. The presence of advance directives may facilitate decision making.

\section{DATA ARCHIVING}

There were no data to deposit.

\section{CONFLICT OF INTEREST}

The authors declare no conflict of interest.

1 Devivo MJ. Epidemiology of traumatic spinal cord injury: trends and future implications. Spinal Cord 2012; 50: 365-372.

2 Casha S, Christie S. A systematic review of intensive cardiopulmonary management after spinal cord injury. J Neurotrauma 2011; 28: 1479-1495.

3 Deyaert J, Chambaere K, Cohen J, Roelands M, Deliens L. Labelling of end-of-life decisions by physicians. J Med Ethics 2014; 40: 505-507.

4 Materstvedt LJ, Clark D, Ellershaw J, Forde R, Gravgaard AM, Muller-Busch HC et al. Euthanasia and physician-assisted suicide: a view from an EAPC Ethics Task Force. Palliat Med 2003; 17: 97-101 (discussion 102-179).

5 Dutch Government. Euthanasia, assisted suicide and non-resuscitation on request. http://www.government.nl/issues/euthanasia/euthanasia-assisted-suicide-and-nonresuscitation-on-request.

6 Buiting H, van Delden J, Onwuteaka-Philpsen B, Rietjens J, Rurup M, van Tol D et al. Reporting of euthanasia and physician-assisted suicide in the Netherlands: descriptive study. BMC Med Ethics 2009; 10: 18.

7 Sise MJ, Sise CB, Thorndike JF, Kahl JE, Calvo RY, Shackford SR. Withdrawal of care: a 10-year perspective at a Level I trauma center. J Trauma Acute Care Surg 2012; 72: 1186-1193.

8 Trunkey DD, Cahn RM, Lenfesty B, Mullins R. Management of the geriatric trauma patient at risk of death: therapy withdrawal decision making. Arch Surg 2000; 135: 34-38.

9 Cooper Z, Rivara FP, Wang J, MacKenzie EJ, Jurkovich GJ. Withdrawal of life-sustaining therapy in injured patients: variations between trauma centers and nontrauma centers. J Trauma 2009; 66: 1327-1335.

10 Franklin GA, Cannon RW, Smith JW, Harbrecht BG, Miller FB, Richardson JD. Impact of withdrawal of care and futile care on trauma mortality. Surgery 2011; 150: 854-860.

11 Ball CG, Navsaria P, Kirkpatrick AW, Vercler C, Dixon E, Zink J et al. The impact of country and culture on end-of-life care for injured patients: results from an international survey. J Trauma 2010; 69: 1323-1333 (discussion 1333-1334).

12 Martin ND, Marks JA, Donohue J, Giordano C, Cohen MJ, Weinstein MS. The mortality inflection point for age and acute cervical spinal cord injury. J Trauma 2011; 71: 380-385 (discussion 385-386).

13 Varma A, Hill EG, Nicholas J, Selassie A. Predictors of early mortality after traumatic spinal cord injury: a population-based study. Spine (Phila Pa 1976) 2010; 35: 778-783.

14 Shao J, Zhu W, Chen X, Jia L, Song D, Zhou X et al. Factors associated with early mortality after cervical spinal cord injury. J Spinal Cord Med 2011; 34: 555-562.

15 Daverat P, Gagnon M, Dartigues JF, Mazaux JM, Barat M. Initial factors predicting survival in patients with a spinal cord injury. J Neurol Neurosurg Psychiatry 1989; 52: 403-406.

16 Selassie AW, Varma A, Saunders LL, Welldaregay W. Determinants of in-hospital death after acute spinal cord injury: a population-based study. Spinal Cord 2013; 51: $48-54$

17 Furlan JC, Bracken MB, Fehlings MG. Is age a key determinant of mortality and neurological outcome after acute traumatic spinal cord injury? Neurobiol Aging 2010; 31: 434-446.

18 Neumann CR, Brasil AV, Albers F. Risk factors for mortality in traumatic cervical spinal cord injury: Brazilian data. J Trauma 2009; 67: 67-70.

19 Claxton AR, Wong DT, Chung F, Fehlings MG. Predictors of hospital mortality and mechanical ventilation in patients with cervical spinal cord injury. Can J Anaesth 1998; 45: 144-149.

20 Patterson DR, Miller-Perrin C, McCormick TR, Hudson LD. When life support is questioned early in the care of patients with cervical-level quadriplegia. $N \mathrm{Engl} \mathrm{J}$ Med 1993; 328: 506-509.

21 Taub AL, Keune JD, Kodner IJ, Schwarze ML. Respecting autonomy in the setting of acute traumatic quadriplegia. Surgery 2014; 155: 355-360.

22 Field HL. A patient with acute traumatic quadriplegia who requested a DNR order. Psychosomatics 2008; 49: 252-254.

23 Nijendijk JH, Post MW, van Asbeck FW. Epidemiology of traumatic spinal cord injuries in The Netherlands in 2010. Spinal Cord 2014; 52: 258-263.

24 World Health Organisation. Definition of palliative care. http://www.who.int/cancer/ palliative/definition/en/.

25 American Association for Automotive Medicine (AAAM). The Abbreviated Injury Scale 1990. Des Plaines, IL, USA.

26 Charlson ME, Pompei P, Ales KL, MacKenzie CR. A new method of classifying prognostic comorbidity in longitudinal studies: development and validation. J Chronic Dis 1987; 40: 373-383.

27 Kirshblum SC, Burns SP, Biering-Sorensen F, Donovan W, Graves DE, Jha A et al. International standards for neurological classification of spinal cord injury (revised 2011). J Spinal Cord Med 2011; 34: 535-546.

28 Gerhart KA, Koziol-McLain J, Lowenstein SR, Whiteneck GG. Quality of life following spinal cord injury: knowledge and attitudes of emergency care providers. Ann Emerg Med 1994; 23: 807-812.

29 Ross LF. Life support for patients with cervical-level quadriplegia. N Engl J Med 1993; 329: 663 (author reply 4). 